\title{
Primary tumor-associated expression of CXCR4 predicts formation of local and systemic recurrency in head and neck squamous cell carcinoma
}

\author{
Andreas Knopf ${ }^{1}$, Leila Bahadori ${ }^{1}$, Kristin Fritsche ${ }^{1}$, Guido Piontek ${ }^{1}$, Cord-Christian \\ Becker $^{1}$, Percy Knolle ${ }^{2}$, Achim Krüger ${ }^{2}$, Henning Bier ${ }^{1}$ and Yin Li ${ }^{1}$ \\ ${ }^{1}$ Otorhinolaryngology, Head and Neck Surgery, Institute of Molecular Immunology \& Experimental Oncology, 81675 München, \\ Germany \\ ${ }^{2}$ Institute of Molecular Immunology \& Experimental Oncology, 81675 München, Germany \\ Correspondence to: Andreas Knopf, email: knopf.ent@gmail.com \\ Keywords: CXCR4; CXCL12; head and neck squamous cell carcinoma; recurrency; metastasis \\ Received: June 23, $2017 \quad$ Accepted: September 30, $2017 \quad$ Published: November 20, 2017 \\ Copyright: Knopf et al. This is an open-access article distributed under the terms of the Creative Commons Attribution License 3.0 \\ (CC BY 3.0), which permits unrestricted use, distribution, and reproduction in any medium, provided the original author and source \\ are credited.
}

\section{ABSTRACT}

Objectives: Despite modern treatment regimens, overall survival in head and neck squamous cell carcinomas (HNSCC) is less than $\mathbf{5 0 \%}$ due to local and systemic disease recurrency. The current study aims to identify molecular markers in primary tumor specimens that predict the risk for local and systemic recurrency at the time of initial diagnosis.

Methods: The study included clinic-pathological data of 1,057 HNSCC. MMP2/9, TIMP1/2, CXCR4, and CXCL12 immunohistochemistry was done in 150 randomly selected specimens. For statistics, we employed Chi square, Fisher exact, and Student's $t$-test. Overall survival (OS) was calculated by Kaplan-Meier and log-rank test. Prognostic variables were subsequently evaluated by Cox regression for forward selection.

Results: CXCR4 positive specimens demonstrated a significant increased risk for tumor recurrency associated death ( $r T: H R$ 10.07; $p=0.001 / r N:$ HR 5.04; $p=0.013$ / rM: HR 2.49; $p=0.029$ ) when compared with their unaltered counterparts. Expression of MMP9, TIMP2, CXCR4, and CXCL12 was significantly increased in distant metastasized patients $(p<0.0001)$ and showed significant cross-correlation. In addition, CXCR4 positivity was associated with an increased risk to die due to enhanced $\mathbf{T}$ or $\mathbf{N}$ status (T1/2 vs. T3/4: HR 5.78; $p=0.017$; NO vs. N+: HR 5.18; $p=0.033$ ).

Conclusion: CXCR4 positivity in tumor samples at initial diagnosis were associated with reduced overall survival, in particular with respect to increasing $\mathrm{T} / \mathrm{N}$ status, local and systemic recurrency.

\section{INTRODUCTION}

Head and neck squamous cell carcinoma (HNSCC) is the sixth most common cancer worldwide accounting for approximately 500,000 newly diagnosed cases and 300,000 deaths every year $[1,2]$. Tobacco abuse and alcohol consumption, as well as infection with human papillomavirus (HPV), were shown to be the most important risk factors in HNSCC carcinogenesis [3, 4]. Beside HPV status, patient's prognosis is inherently associated with the T, N, M status [5,
6]. Tumor stage-adjusted pre-therapeutic staging determines resectability at primary tumor site and cervical lymph node basin and excludes distant metastasis [7]. Local lymph node metastasis occurs in approximately $60 \%$ of HNSCC cases, while distant metastases can be diagnosed in $5 \%$ at initial diagnosis $[6,8]$. In the absence of distant metastasis, surgery with/without adjuvant (chemo-) radiation and primary chemo-radiation represent the therapeutic mainstay of curative approaches $[5,9]$. Curative treatment has to manage the balancing act between sufficient radicality and 
preservation of functional structures [10-12]. Both, surgical and conservative approaches have been associated with severe comorbidity [10-13]. Despite significant advances in cancer treatment, the 5-year survival rate is less than 50\% due to both local relapse and development of distant metastases [4]. While a substantial proportion of HNSCC patients suffer from relapse at primary tumor site and/or cervical lymph nodes, further $15-20 \%$ of all HNSCC develop metachronous distant metastases after primary curative treatment, and therefore, become palliative $[5,9]$. Thus, it is important to aim for identification of factors allowing prediction of disease recurrence already at the time of initial diagnosis [14]. We recently demonstrated in colorectal cancer that CXCL12 seems to be a decisive factor in the establishment of a pre-metastatic niche in the liver and interference with the CXCL12-CXCR4 axis may inhibit the pre-deposition of a distant organ to attract metastases [15]. In HNSCC, CXCR4 positivity resulted in reduced overall survival (OS) in CXCR4 positive specimens [16]. Molecular mechanisms underlying the worse survival in HNSCC, particularly with respect to tumor recurrency, remain unclear. This prompted us to investigate the impact of proteins associated with the CXCR4-CXCL12 axis (MMP2, MMP9, TIMP1, TIMP2, CXCR4, and CXCL12) in local recurrency and distant metastatic spread of HNSCC. Increased CXCR4 levels correlated with the development of local recurrent disease and distant metastases in HNSCC resulting in a dramatically decreased overall survival.

\section{RESULTS}

\section{Patient and tumor characteristics}

The investigated cohort of 1,057 consecutively surgically and conservative treated HNSCC patients included 841 men and 216 women with a median and mean age of 60 years. The majority of tumors originated in the oropharynx, followed by hypopharynx, larynx, and the oral cavity. While $60 \%$ of patients demonstrated lymph node metastasis at the time of diagnosis, M1 status was present in $4 \%$, only. Almost $66 \%$ of our patients underwent head and neck surgery with/without adjuvant chemo-radiation. Ninety percent of tumors were assumed to be resected in sano, comprising $\mathrm{R} 0$ resection in $87 \%$ and piecemeal resection $(\mathrm{Rx})$ in $7 \%$ of patients. Thirty-four percent of our patients were treated by primary radio(chemo-)therapy (Table 1).

\section{CXCR4 positivity is associated with local and systemic disease recurrence}

Patients who develop local and/or systemic recurrency demonstrated a significant reduced OS (Figure 1A). While patients without recurrent disease showed a mean OS of 94 months, survival in individuals suffering from recurrency was 38 months (Figure 1A). Subgroup analysis of recurrent disease localization demonstrated a mean OS in patients with rT0 status of 92 months and decreased by recurrency at primary tumor site $(\mathrm{rT}+)$ to 44 months $(p<0.0001$; Figure $1 B)$. OS in patients with recurrency in local lymph nodes $(\mathrm{rN}+)$ was 47 months, while mean OS was 76 months in unaltered counterparts $(\mathrm{p}<0.0001$; Figure 1C). Patients with systemic tumor recurrency $(\mathrm{rM}+)$ showed the lowest overall survival with 25 months, while patients without systemic disease showed a mean overall survival of 88 months $(p<0.0001$; Figure 1D). Subgroup analysis of different combination of tumor recurrency (rT $+\mathrm{rNOrM0}$ vs. $\mathrm{rTOrN}+\mathrm{rM} 0$ vs. rTOrN0rM+ vs. $r \mathrm{~T}+\mathrm{rN}+\mathrm{rM0}$ vs. $\mathrm{rT}+\mathrm{rNOrM}+\mathrm{vs}$. $\mathrm{rT}+\mathrm{rN}+\mathrm{rM}+$ ) did not reveal differences between the groups $(\mathrm{p}=0.57$; data not shown). Forward selected, proportional Cox regression of survival modifying parameters $(T, N$, R, G, tumor localization, MMP2/9, TIMP1/2, CXCR4, and CXCL12) identified CXCR4 being the only survivalmodifying parameter in HNSCC (Table 2). CXCR4 positivity at primary tumor site was associated with a significantly increased risk for recurrency associated death ranging from $\mathrm{HR}=2.49$ in patients with systemic disease recurrency, $\mathrm{HR}=5.04$ in patients with local lymph node recurrency to HR: 10.07 in patients with recurrency at primary tumor site (Table 2). In addition, MMP9-positive patients showed an increased risk for $\mathrm{rN}+$-associated death (HR: 2.25; Table 2). Interestingly, multivariate analysis failed to identify $\mathrm{N}+$ and increasing $\mathrm{T}$ status, which traditionally were held to be responsible for local and systemic recurrency-associated death, as independent risk factors. Furthermore, forward-selected proportional cox regression analyzing reduced survival in advanced (T1/2 vs. T3/4) or lymph node positive HNSCC (N0 vs. N+) also demonstrated CXCR4 as solitary risk factor (Table 2). Proportional Cox regression model estimated HR $=5.78$ and $\mathrm{HR}=5.18$ deaths due to increased T status (T1/2 vs. $\mathrm{T} 3 / 4 ; \mathrm{p}=0.017$; Table 2 ) and lymph node positivity (N0 vs. $\mathrm{N}+; \mathrm{p}=0.033$; Table 2). Therefore, patients with advanced $\mathrm{T}$ status (T3/4) and lymph node positivity demonstrated significant worse survival when compared with $\mathrm{T} 1 / 2$ $(p<0.0001$; Figure 1E) and N0 ( $=0.02$; Figure $1 F)$ status.

\section{CXCR4 positivity is associated with the development of distant metastasis}

After pre-therapeutic staging, $96 \%$ of our patients were held to be distant metastasis free, and therefore, underwent curative treatment (Table 1). After a mean follow-up time of 18 months, $14 \%$ of these patients were diagnosed with metachronous distant metastasis. The vast majority of patients $(82 \%)$ showed single organ manifestation, the lungs being the preferential organ of distant metastatic outgrowth (Table 3).

Immunohistochemistry of CXCR4-CXCL12 axisassociated proteins revealed significantly higher expression of MMP9, TIMP2, CXCR4, and CXCL12 in distant metastasized specimens as compared to their unaltered 
Table 1: Epidemiologic and tumor characteristics demonstrate a typical HNSCC cohort with pronounced tumor occurrence in male at $6^{\text {th }}$ decade of life

\begin{tabular}{|c|c|}
\hline & HNSCC \\
\hline $\mathbf{N}$ & 1057 \\
\hline \multicolumn{2}{|l|}{ Age (years) } \\
\hline Median $[25 \% ; 75 \%]$ & $60[53 ; 67]$ \\
\hline Mean \pm SD & $60 \pm 11$ \\
\hline \multicolumn{2}{|l|}{ Sex, n (\%) } \\
\hline Male & $841(80)$ \\
\hline Female & $216(20)$ \\
\hline \multicolumn{2}{|l|}{ Location } \\
\hline Sinunasal & $35(3)$ \\
\hline Nasopharynx & $20(2)$ \\
\hline Oropharynx & $415(39)$ \\
\hline Hypopharynx & $220(21)$ \\
\hline Larynx & $208(20)$ \\
\hline Oral cavity & $154(15)$ \\
\hline CUP & 5 \\
\hline \multicolumn{2}{|l|}{ T status, n (\%) } \\
\hline $\mathrm{Tx}$ & 8 \\
\hline $\mathrm{T} 1$ & $276(26)$ \\
\hline $\mathrm{T} 2$ & $313(30)$ \\
\hline $\mathrm{T} 3$ & $221(21)$ \\
\hline $\mathrm{T} 4$ & $239(23)$ \\
\hline \multicolumn{2}{|l|}{ N status, n (\%) } \\
\hline No & $417(40)$ \\
\hline $\mathrm{N}+$ & $640(60)$ \\
\hline \multicolumn{2}{|l|}{ M status, n (\%) } \\
\hline M0 & $1015(96)$ \\
\hline M1 & $42(4)$ \\
\hline \multicolumn{2}{|l|}{ Grading, n (\%) } \\
\hline G1 & $45(4)$ \\
\hline G2 & $506(48)$ \\
\hline G3 & $469(45)$ \\
\hline G4 & $13(1)$ \\
\hline Gx & $24(2)$ \\
\hline \multicolumn{2}{|l|}{ R status, n (\%) } \\
\hline $\mathrm{R} 0$ & $590(83)$ \\
\hline $\mathrm{R} 1$ & $58(8)$ \\
\hline
\end{tabular}




\begin{tabular}{lc}
\hline & HNSCC \\
\hline R2 & $14(1)$ \\
Rx & $50(7)$ \\
Treatment, n (\%) & \\
OP only & $187(19)$ \\
OP + RTX & $300(28)$ \\
OP + RCTX & $203(19)$ \\
Prim. RCTX & $329(31)$ \\
Prim. RTX & $36(3)$ \\
\hline
\end{tabular}

Tumors were frequently localized in the oropharynx, hypopharynx, larynx, and oral cavity. While $60 \%$ of patients showed lymph node metastasis at the time of diagnosis, distant metastasis was diagnosed in $4 \%$. OP=surgical resection, RTX=radiotherapy, $\mathrm{RCTX}=$ radio-chemotherapy.

Table 2: Cox regression of recurrency and $\mathrm{T} / \mathrm{N}$ status modifying parameters

\begin{tabular}{lccc}
\hline & HR & $\mathbf{9 5 \% - C I}$ & p-value \\
\hline rT0 vs. $\mathbf{r T}+$ & & & 0.001 \\
CXCR4 pos. & 10.07 & $9.78-11.02$ & \\
rN0 vs. rN+ & & & 0.013 \\
CXCR4 pos. & 5.04 & $4.48-5.24$ & 0.048 \\
MMP9 pos. & 2.25 & $2.02-2.51$ & \\
rM0 vs. rM+ & & & 0.029 \\
CXCR4 pos. & 2.49 & $1.10-5.55$ & \\
T1/2 vs. T3/4 & & & 0.017 \\
CXCR4 pos. & 5.78 & $2.44-6.01$ & \\
N0 vs. $\mathbf{N}+$ & & & 0.033 \\
CXCR4 pos. & 5.18 & $2.40-6.00$ & \\
\hline
\end{tabular}

Forward selected proportional Cox regression of 150 randomly selected patients corresponded to survival curves of the entire cohort (Figure 1A-1F). CXCR4 was the only disease modifying parameter. While increasing $\mathrm{T}$ and $\mathrm{N}$ status failed to be a prognostic parameter in tumor recurrency, subgroup analysis of different $\mathrm{T}$ and $\mathrm{N}$ status also identified CXCR4 being disease modifying.

counterparts (Figure 2). Although FFPE samples were randomly selected from a huge cohort of consecutively included patients with different tumor localization and TNM status, statistical analysis still revealed significant correlation between CXCR4, MMP2 ( $\mathrm{r}=0.2 ; \mathrm{p}=0.04)$, TIMP1 ( $\mathrm{r}=0.32 ; \mathrm{p}=0.001)$, TIMP2 $(\mathrm{r}=0.66 ; \mathrm{p}<0.0001)$, and CXCL12 (r=0.53; $<<0.0001)$ (Table 4).

\section{DISCUSSION}

Head and neck surgery and primary radio(chemo-) therapy represent therapeutic state-of-the-art in curative HNSCC treatment $[5,7]$. Both therapeutic regimens aim to achieve sufficient radicality with a minimum of functional loss. However, all curative approaches are associated with severe patient's morbidity $[10,13]$. The current cohort includes a typical HNSCC profile concerning age/gender distribution, primary tumor localization, and TNM status. Therefore, the vast majority of patients (96\%) underwent curative treatment. Despite significant advances in HNSCC treatment, the 5-year survival rate is less than 50\% due to both local relapse and the development of distant metastases [4]. Tumor stage adjusted pre-therapeutic imaging validates operability at primary tumor site and local lymph nodes and excludes distant metastatic spread. Pre-therapeutic thoracic computed tomography, which was applied in the majority of our patients due to advanced disease status, showed distant metastatic outgrowth in only $4 \%$ of patients at the time of diagnosis. After a mean time of 18 months, another $14 \%$ of our patients developed 

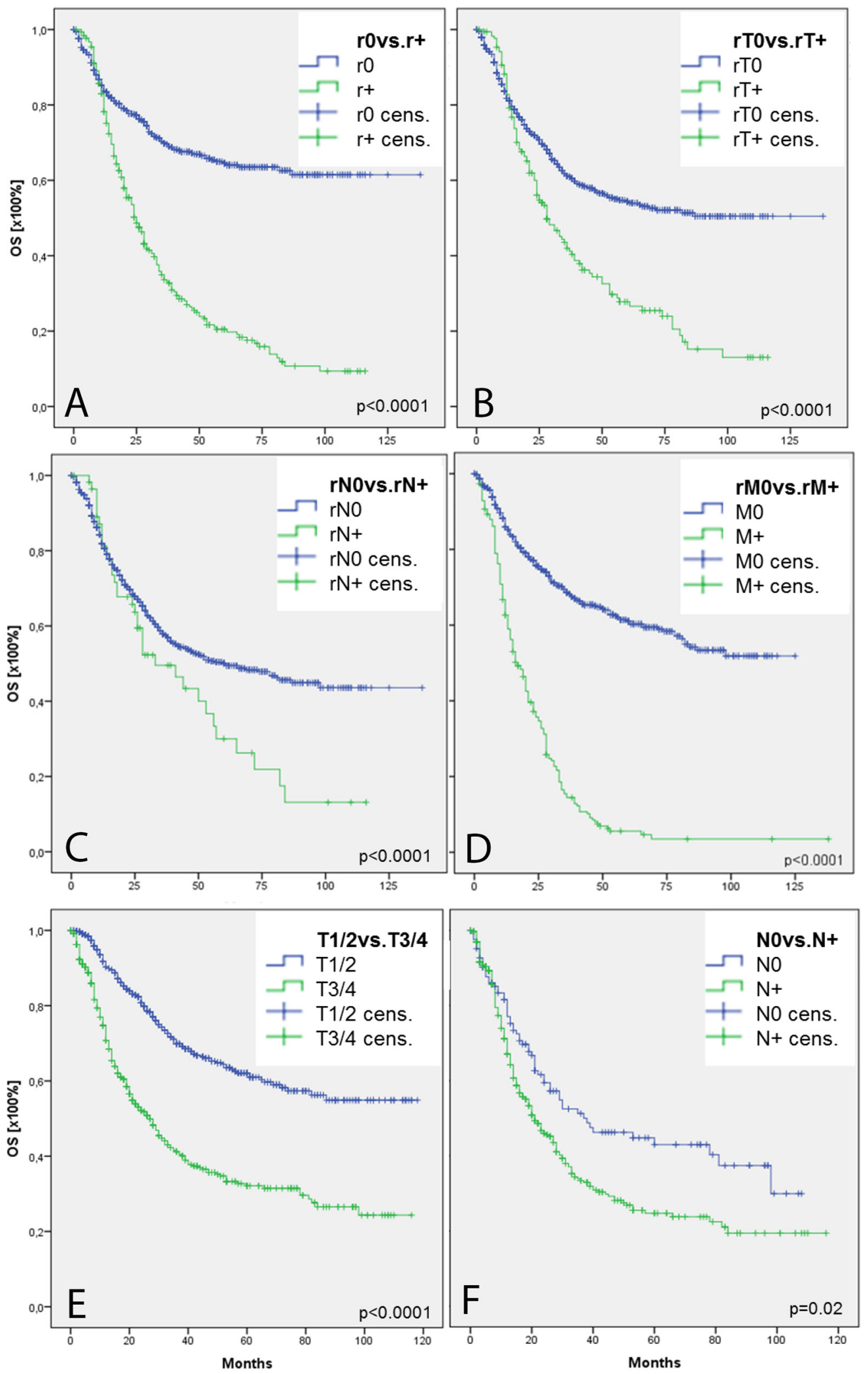

Figure 1: Overall survival in HNSCC. Kaplan Maier illustrates overall survival in 1,057 HNSCC patients with respect to tumor recurrency (A). Subgroup analysis demonstrates survival curve in patients with/without recurrency at primary tumor site (B), regional lymph nodes (C), and distant metastasis (D). Fugures (E and F) show survival curves in patients with limited (T1/2 or N0) and aggravated (T3/4 or $\mathrm{N}+$ ) disease burden. 
Table 3: Distant metastatic profile

HNSCC

\begin{tabular}{lc}
\hline M stage, n (\%) & $42(4)$ \\
M1 synchronous & $143(14)$ \\
M1 metachronous & \\
Time frame, month \\
(mean \pm SD) \\
Diagnosis to \\
metachronous M1 \\
Location, n (\%) \\
Multi-organ disease \\
Liver \\
Lung \\
Mediastinum \\
Bone & $34(18)$ \\
Skin & $34(18)$ \\
Central nervous system & $12(7)$ \\
Peripheral lymph nodes & $32(17)$ \\
Other & $16(9)$ \\
\hline
\end{tabular}

Distant metastasis was diagnosed in $4 \%$ at initial diagnosis. After a mean time of 18 months, metachronous distant metastasis occurred in 14\%. Lungs were predominantly affected. metachronous distant metastatic outgrowth, in particular pulmonary metastases. The diagnostic gap in the pretherapeutic identification of pulmonary metastases was recently demonstrated [17] and highlights the necessity to identify molecular markers that predict both, local and systemic tumor recurrency at the time of initial diagnosis. Recently, CXCR4 came into focus in different tumor entities and CXCR4-positivity was usually associated with local tumor growth, the occurrence of local and systemic metastases and, therefore, reduced survival parameters [14]. However, little is known about the impact of CXCR4-CXCL12 axis in HNSCC. This prompted us to investigate molecular aspects in cohort of 1,057 HNSCC patients with respect to local and systemic tumor disease recurrency as most important indicator in therapy failure. In agreement with Zhao et al. the current study identified CXCR4 as an important survival-associated parameter in HNSCC [16]. CXCR4 positivity of the primary tumor at the time of diagnosis was associated with significantly increased risk for local and systemic recurrency-associated death. Interestingly, advanced T- and N-status did not directly correlate in reduced OS due to development of distant metastasis, but rather due to local tumor progression (T1/2 vs. T3/4). In addition, development of regional metastases ( $\mathrm{N} 0$ vs. $\mathrm{N}+$ ) was associated with the CXCR4 status. These results are in agreement with Ishikawa et al. who demonstrate a correlation between CXCR4 expression and development of lymph node metastasis in 90 HNSCC patients [18]. Above that, we could now demonstrate that CXCL12 positivity at primary tumor site was associated with a significant increased



Figure 2: Immunohistochemical expression of markers associated with the CXCR4 axis. MMP9, TIMP2, CXCR4, and CXCL12 were significantly higher expressed in distant metastasized patients when compared with their unaltered counterparts. 
Table 4: Correlation of immunohistochemical markers

\begin{tabular}{lccccccc}
\hline & & MMP2 & MMP9 & TIMP1 & TIMP2 & CXCR4 & CXCL12 \\
\hline \multirow{2}{*}{ MMP2 } & r-value & 1 & 0.003 & 0.12 & 0.11 & $\mathbf{0 . 2}$ & $\mathbf{0 . 2 3}$ \\
& p-value & & 0.98 & 0.2 & 0.28 & $\mathbf{0 . 0 4}$ & $<\mathbf{0 . 0 2}$ \\
MMP9 & r-value & 0.003 & 1 & 0.004 & $\mathbf{0 . 2 7}$ & 0.12 & 0.12 \\
& p-value & 0.98 & & 0.97 & $\mathbf{0 . 0 0 4}$ & 0.23 & 0.2 \\
TIMP1 & r-value & 0.12 & 0.004 & 1 & $\mathbf{0 . 2 4}$ & $\mathbf{0 . 3 2}$ & $\mathbf{0 . 3 1}$ \\
& p-value & 0.2 & 0.97 & & $\mathbf{0 . 0 1}$ & $\mathbf{0 . 0 0 1}$ & $\mathbf{0 . 0 0 1}$ \\
TIMP2 & r-value & 0.11 & $\mathbf{0 . 2 7}$ & $\mathbf{0 . 2 4}$ & 1 & $\mathbf{0 . 6 6}$ & $\mathbf{0 . 5 2}$ \\
& p-value & 0.28 & $\mathbf{0 . 0 0 4}$ & $\mathbf{0 . 0 1}$ & & $<\mathbf{0 . 0 0 0 1}$ & $<\mathbf{0 . 0 0 0 1}$ \\
CXCR4 & r-value & $\mathbf{0 . 2}$ & 0.12 & $\mathbf{0 . 3 2}$ & $\mathbf{0 . 6 6}$ & 1 & $\mathbf{0 . 5 3}$ \\
& p-value & $\mathbf{0 . 0 4}$ & 0.23 & $\mathbf{0 . 0 0 1}$ & $<\mathbf{0 . 0 0 0 1}$ & & $<\mathbf{0 . 0 0 0 1}$ \\
CXCL12 & r-value & $\mathbf{0 . 2 3}$ & 0.12 & $\mathbf{0 . 3 2}$ & $\mathbf{0 . 5 2}$ & $\mathbf{0 . 5 3}$ & 1 \\
& p-value & $<\mathbf{0 . 0 2}$ & 0.2 & $\mathbf{0 . 0 0 1}$ & $<\mathbf{0 . 0 0 0 1}$ & $<\mathbf{0 . 0 0 0 1}$ & \\
\hline
\end{tabular}

150 randomly selected samples of consecutively included patients showed significant correlation between CXCR4, MMP9, TIMP1, TIMP2, and CXCL12 suggesting functional significance.

risk of lymph node recurrency associated death. In oral cancer, a significantly higher CXCL12 expression was observed in lymph node metastases when compared with the corresponding primary tumor specimens, raising the hypothesis that CXCR4 might be attracted in a paracrine manner on the basis of the CXCL12 gradient from primary tumor towards lymph nodes [19]. More recently, functional aspects of the CXCR4-CXCL12 axis were demonstrated in the establishment of a pre-metastatic niche in the liver [15]. In the current study, protein expression of MMP9, CXCR4, CXCL12, and TIMP2 was significantly higher in distant metastasized individuals when compared with their unaltered counterparts. Although analyzed specimens were randomly selected from a cohort of 1,057 consecutively treated patients, immunohistochemistry revealed correlation between CXCR4, CXCL12, MMP9, TIMP1, and TIMP2. These results agree with the current literature that demonstrated a CXCL12-dependent increase of MMP2 and MMP9 secretion via activation of the ERK$1 / 2$ signaling pathway [20,21]. The association of these markers with the occurrence of distant metastases has already been shown in other tumor entities [19, 22-24]. However, molecular mechanisms of local and systemic disease recurrency in HNSCC mediated by the CXCR4CXCL12 axis are still delusive at this point.

\section{MATERIALS AND METHODS}

\section{Patient selection}

The current study includes a total of 1,057 HNSCC patients who were consecutively diagnosed in the ENT department of the University Hospital Rechts der Isar, Munich. Tumor samples were histologically reviewed by at least two experienced pathologists. Dysplasia, carcinoma in situ, and other histologic subtypes were excluded. Clinical parameters and survival data were retrospectively collected: age, sex, TNM status ( $7^{\text {th }}$ edition), grading, treatment modalities, recurrence, and death/loss to follow-up. Patients with lacking data, incomplete staging, and refused/ not finished surgical and/or conservative treatment were excluded from survival analysis. The mean follow up time was $\geq 60$ months for all analyzed tumor entities.

\section{Immunohistochemistry}

HNSCC tumor samples were obtained from primary tumor sites at the time of diagnosis. Paraffinembedded tumor (FFPE) samples from 150 HNSCC were randomly selected from the overall cohort and analyzed via immunohistochemistry (IHC). Subgroup analysis excluded study population driven bias $(\mathrm{p}=0.16-0.93)$. FFPE tumor sections $(2.5 \mu \mathrm{m})$ were MMP2 (DCS Innovative DiagnostikSysteme, Hamburg, Germany, 1:100), MMP9 (Biomol GmbH, Hamburg, Germany, 1:1000), TIMP1 (R\&D, Wiesbaden, Germany, 1:500), TIMP2 (Biomol, 1:500), CXCR4 (R\&D, 1:200), and CXCL12 (R\&D, 1:1000) immuno-stained and visualized with the Bond Polymer Refine Detection Kit (Leica, Nussloch, Germany). Cytoplasmatic expression levels were classified using a scoring system analyzing the staining intensity $(0=$ no staining, $1=$ low, $2=$ moderate, $3=$ strong staining intensity) and the relative proportion of stained tumor cells $(0$, $1=<10 \%, 2=10-39 \%, 3=40-69 \%, 4=>70$ of the tumor cells). A cumulative score (range 0-7 points) was assessed by adding 
both scores. A positive staining was defined by a cumulative score equal or greater than 3 .

\section{Statistical analysis}

Differences between the groups were analyzed using the Chi square test and Fisher exact test for categorical, and the unpaired student's $t$-test for continuous variables. Correlation between different markers were calculated and expressed by Pearson's r. As main endpoint the overall survival (OS) was assessed measuring the time from treatment to death of any cause. Survival rates and curves were calculated and illustrated by the KaplanMeier method and further analyzed by the log-rank test. Variables that revealed prognostic or effect modifying potential on the outcome were subsequently evaluated by the proportional Cox regression for forward selection. p-values $<0.05$ were considered statistically significant. Statistical analysis was done using SPSS (SPSS Inc., Chicago, IL).

\section{CONCLUSIONS}

CXCR4 positivity in HNSCC is associated with increased risk of local and systemic recurrency associated death. The increased risk can be identified by CXCR4 over-expression at primary tumor site, providing a diagnostic approach to improve treatment stratification.

\section{CONFLICTS OF INTEREST}

All authors state no conflicts of interest.

\section{FINANCIAL DISCLOSURE}

All authors state no financial disclosures.

\section{REFERENCES}

1. Jemal A, Bray F, Center MM, Ferlay J, Ward E, Forman D. Global cancer statistics. CA Cancer J Clin. 2011; 61:69-90.

2. Siegel RL, Miller KD, Jemal A. Cancer statistics 2016. CA Cancer J Clin. 2016; 66:7-30.

3. Murata M, Takayama K, Choi BC, Pak AW. A nested casecontrol study on alcohol drinking, tobacco smoking, and cancer. Cancer Detect Prev. 1996; 20:557-565.

4. Forastiere A, Koch W, Trotti A, Sidransky D. Head and neck cancer. N Engl J Med. 2001; 345:1890-1900.

5. Haigentz M Jr, Hartl DM, Silver CE, Langendijk JA, Strojan P, Paleri V, de Bree R, Machiels JP, Hamoir M, Rinaldo A, Paccagnella D, Shaha AR, Takes RP, et al. Distant metastases from head and neck squamous cell carcinoma. Part III. Treatment. Oral Oncol. 2012; 48:787-793.

6. Jacobi C, Ayx I, Fritsche K, Piontek G, Hoffmann D, Weirich G, Knopf A. Potential impact of human papilloma virus on survival of basaloid squamous carcinoma of the head and neck. Oncotarget. 2015; 6:3462-3470. https://doi. org/10.18632/oncotarget.3062.

7. Sadick M, Schoenberg SO, Hoermann K, Sadick H. Current oncologic concepts and emerging techniques for imaging of head and neck squamous cell cancer. GMS Curr Top Otorhinolaryngol Head Neck Surg. 2012; 11:Doc08.

8. Kotwall C, Sako K, Razack MS, Rao U, Bakamjian V, Shedd DP. Metastatic patterns in squamous cell cancer of the head and neck. Am J Sur. 1987; 154:439-442.

9. Bhave SL, Teknos TN, Pan Q. Molecular parameters of head and neck cancer metastasis. Crit Rev Eukaryot Gene Expr. 2011; 21:143-153.

10. Baddour HM Jr, Magliocca KR, Chen AY. The importance of margins in head and neck cancer. J Surg Oncol. 2016; 113:248-255.

11. Knopf A, Mansour N, Hofauer B, Bier H, Scherer EQ. Outcome after pharyngeal reconstruction using pectoralis major and radial forearm flap after resection of pharyngeal and laryngeal squamous cell carcinomas. Eur Arch Otorhinolaryngol. 2016; 273:2637-2642.

12. Teutsch S, Bas M, Bier H, Knopf A. [Stomal Cancer Recurrency, A Clinic-Pathological Consideration]. [Article in German]. Laryngorhinootologie. 2017; 96:239-243.

13. Psyrri A, Licitra L, Lacombe D, Schuuring E, Budach W, Ozsahin M, Knecht R, Vermorken JB, Langendijk JA. Strategies to promote translational research within the European Organisation for Research and Treatment of Cancer (EORTC) Head and Neck Cancer Group: a report from the Translational Research Subcommittee. Ann Oncol. 2010; 21:1952-1960.

14. Knopf A, Fritsche MK, Li Y. CXCR4: A New Player in an Old Scene? ORL J Otorhinolaryngol Relat Spec. 2017; 79:34-42.

15. Seubert B, Grünwald B, Kobuch J, Cui H, Schelter F, Schaten S, Siveke JT, Lim NH, Nagase H, Simonavicius N, Heikenwalder M, Reinheckel T, Sleeman JP, et al. Tissue inhibitor of metalloproteinases (TIMP)-1 creates a premetastatic niche in the liver through SDF-1/CXCR4-dependent neutrophil recruitment in mice. Hepatology. 2015; 61:238-248.

16. Zhao H, Guo L, Zhao H, Zhao J, Weng H, Zhao B. CXCR4 over-expression and survival in cancer: a system review and meta-analysis. Oncotarget. 2015; 6:5022-5040. https://doi. org/10.18632/oncotarget.3217.

17. Kwon M, Lee SH, Lee YS, Lee CW, Roh JL, Choi SH, Nam SY, Kim SY. Clinical implications of nonspecific pulmonary nodules identified during the initial evaluation of patients with head and neck squamous cell carcinoma. Eur Radiol. 2017; 27:3725-3732.

18. Ishikawa T, Nakashiro K, Hara S, Klosek SK, Li C, Shintani $\mathrm{S}$, Hamakawa H. CXCR4 expression is associated with lymph-node metastasis of oral squamous cell carcinoma. Int J Oncol. 2006; 28:61-66.

19. Uchida D, Onoue T, Tomizuka Y, Begum NM, Miwa Y, Yoshida H, Sato M. Involvement of an autocrine stromal 
cell derived factor-1/CXCR4 system on the distant metastasis of human oral squamous cell carcinoma. Mol Cancer Res. 2007; 5:685-694.

20. Samara GJ, Lawrence DM, Chiarelli CJ, Valentino MD, Lyubsky S, Zucker S, Vaday GG. CXCR4-mediated adhesion and MMP-9 secretion in head and neck squamous cell carcinoma. Cancer Lett. 2004; 214:231-241.

21. Janowska-Wieczorek A, Matsuzaki A, Marquez A. The Hematopoietic Microenvironment: Matrix Metalloproteinases in the Hematopoietic Microenvironment. Hematology. 2000; 4:515-527.

22. Mendes O, Kim HT, Stoica G. Expression of MMP2, MMP9 and MMP3 in breast cancer brain metastasis in a rat model. Clin Exp Metastasis. 2005; 22:237-246.
23. Lim JB, Chung HW. Serum ENA78/CXCL5, SDF-1/ CXCL12, and their combinations as potential biomarkers for prediction of the presence and distant metastasis of primary gastric cancer. Cytokine. 2015; 73:16-22.

24. Fuksiewicz M, Kotowicz B, Rutkowski A, Kowalska M. The matrix metalloproteinase-7 and pro-enzyme of metalloproteinase- 1 as a potential marker for patients with rectal cancer without distant metastasis. Tumour Biol. 2015; 36:3629-3635. 